\title{
A New Relaxed State in Horse Methemoglobin Characterized by Crystallographic Studies
}

\author{
R. Sankaranarayanan, B.K. Biswal, and M. Vijayan* \\ Molecular Biophysics Unit, Indian Institute of Science, Bangalore, India
}

\begin{abstract}
A new relaxed state has been characterized in the crystals of horse methemoglobin grown at neutral pH at low ionic concentration and their low humidity variants. The crystals provide an example for improvement in X-ray diffraction quality with reduced solvent content. Only the classical $R$ state has been so far observed in liganded horse hemoglobin. The state characterized in the present study lies in between the $R$ state and the $R 2$ state characterized earlier in liganded human hemoglobin. The results presented here, along with those of earlier studies, suggest that relaxed and tense hemoglobin can access ensembles of states. Proteins 2005; 60:547-551. ๑ 2005 Wiley-Liss, Inc.
\end{abstract}

Key words: hemoglobin; X-ray crystallography; quaternary association; protein hydration; intersubunit mobility; allosteric transition

\section{INTRODUCTION}

Hemoglobin, along with myoglobin, is the first protein to be structure analyzed using X-ray crystallography. Thanks particularly to the monumental work of Perutz, ${ }^{1}$ it is also among the most thoroughly studied proteins. It has been the best example and model for allosteric transitions in proteins. In conformity with the suggestions of Monod, Wyman, and Chengeux, ${ }^{2}$ tetrameric hemoglobin exists in two multimeric states, namely, the relaxed liganded $\mathrm{R}$ state and the tense unliganded $\mathrm{T}$ state. The metal ion in heme group is ferrous in both the states. The nonfunctional methemoglobin with a ferric ion instead of a ferrous ion in the heme also exists in the $R$ state. Except for differences in the immediate vicinity of the heme group, particularly the metal ion, the molecule in both the states has nearly the same tertiary structure. The difference between the two states is primarily in the quaternary association.

Although most of the original definitive results were obtained using horse hemoglobin, the protein from other sources also received considerable attention. ${ }^{3}$ In particular, much of the work in recent years has been focused on human hemoglobin. ${ }^{4-9}$ An interesting result to emanate from studies on the human protein was the identification of a new relaxed state, designated $\mathrm{R} 2,{ }^{10}$ which has quaternary structure substantially different from that of the protein in the $\mathrm{R}$ state. ${ }^{4}$ The $\mathrm{R} 2$ state was observed in crystals grown in a medium with lower ion concentration as well as $\mathrm{pH}$ than the medium from which the crystals containing R-state hemoglobin were grown. Subsequently, we analyzed crystals containing three crystallographically independent tetrameric molecules, of human methemoglobin from a medium with comparatively low ionic concentration, but high $\mathrm{pH} .{ }^{11}$ The three molecules in these crystals have quaternary structures intermediate between those of $\mathrm{R}$ and R2 states. Thus it appeared that hemoglobin can access different relaxed states with varying degrees of similarity among them. A similar conclusion was arrived at in a parallel study involving bovine carbonmonoxyhemoglobin. ${ }^{12}$ Subsequently, we demonstrated through crystallographic studies of a low humidity crystal form of human deoxyhemoglobin, ${ }^{13}$ that the tense state can also exist in an ensemble of structures.

Although much of the fundamental results on hemoglobin were derived from the equine protein, the R2 state has not been characterized in the crystals of horse hemoglobin. The structure of a crystal of horse methemoglobin obtained at low $\mathrm{pH}$ (5.4) was determined with a view to characterize this state, but it was found that the molecules in the crystal exist in the $\mathrm{R}$ state. ${ }^{14}$ Thus, the variability of the relaxed state of horse hemoglobin merits further exploration. In this context, here we report the structure of a horse methemoglobin crystal form grown using polyethelene glycol at comparatively high $\mathrm{pH}$ (7.0). The results provide insights into the structural variability of relaxed state horse hemoglobin. Incidentally, the present work also provides an example of the improvement in the quality of the diffraction data accompanying reduction in solvent content.

\section{MATERIALS AND METHODS}

Horse methemoglobin was purchased from Sigma Chemical Company. A $25 \mathrm{mg} / \mathrm{ml}$ solution of the protein in $0.01 \mathrm{M}$ ammonium phosphate buffer, $\mathrm{pH}$ 7.0, was first dialyzed against the same buffer using a $12 \mathrm{KDa}$ dialysis membrane sack to remove impurities. The dialyzed protein solution was mixed with twice the volume of a $30 \%(\mathrm{w} / \mathrm{v})$ solution of PEG 3350. Crystals grew in a week. The crystal was maintained at a relative humidity (r.h) of $98 \%$ by placing a drop of a saturated solution of $\mathrm{CuSO}_{4} \cdot 5 \mathrm{H}_{2} \mathrm{O}$ approximately $1 \mathrm{~cm}$ from the crystal in the capillary before

\footnotetext{
*Correspondence to: Molecular Biophysics Unit, Indian Institute of Science, Bangalore 560 012, India. E-mail: mv@mbu.iisc.ernet.in

Received 8 September 2004; Revised 21 December 2004; Accepted 28 January 2005

Published online 10 May 2005 in Wiley InterScience (www.interscience.wiley.com). DOI: 10.1002/prot.20510
} 
TABLE I. Data Collection and Refinement Statistics

\begin{tabular}{|c|c|c|c|}
\hline & Native & r.h. $98 \%$ & r.h. $88 \%$ \\
\hline Space group & $\mathrm{P} 2_{1} 2_{1} 2_{1}$ & $\mathrm{P} 2_{1} 2_{1} 2_{1}$ & $\mathrm{P} 2_{1} 2_{1} 2_{1}$ \\
\hline $\mathrm{a}(\AA)$ & 63.30 & 62.80 & 62.52 \\
\hline $\mathrm{b}(\AA)$ & 83.07 & 80.91 & 80.14 \\
\hline$c(\AA)$ & 112.09 & 112.16 & 111.32 \\
\hline $\mathrm{Z}$ & 4 & 4 & 4 \\
\hline Unit cell Volume $\left(\AA^{3}\right)$ & 589413 & 569857 & 557718 \\
\hline Solvent content $(\%)$ & 46.1 & 44.3 & 43.1 \\
\hline Data resolution $(\AA)$ & 3.1 & 2.6 & 2.3 \\
\hline Last Shell $(\AA)$ & $3.21-3.1$ & $2.69-2.6$ & $2.38-2.3$ \\
\hline Number of measured reflections & 30099 & 69356 & 115564 \\
\hline Number of Unique reflections & $10748(1028)$ & $18160(1778)$ & $24502(2373)$ \\
\hline Completeness $(\%)$ & $95.9(93.5)$ & $99.9(100)$ & $96.0(94.9)$ \\
\hline $\mathrm{R}_{\text {merge }}^{\mathrm{a}}(\%)$ & $18.8(51.1)$ & $14.3(47.4)$ & $11.2(52.3)$ \\
\hline Resolution limits used in refinement $(\AA)$ & $20.0-3.1$ & $20.0-2.6$ & $20.0-2.3$ \\
\hline Number of reflections with $\mathrm{F}>0$ & 10174 & 17215 & 24883 \\
\hline $\mathrm{R}$-factor ${ }^{\mathrm{b}}(\%)$ & 20.1 & 16.7 & 18.3 \\
\hline $\mathrm{R}_{\text {free }} \mathrm{b}(\%)$ & 27.4 & 22.8 & 22.8 \\
\hline Protein atoms & 4406 & 4406 & 4406 \\
\hline Solvent atoms & 136 & 304 & 386 \\
\hline ligand atoms & 172 & 172 & 172 \\
\hline \multicolumn{4}{|l|}{ RMS deviation from ideal values } \\
\hline Bond lengths $(\AA)$ & 0.010 & 0.009 & 0.008 \\
\hline Bond angles $\left({ }^{\circ}\right)$ & 1.4 & 1.3 & 1.2 \\
\hline Dihedral angles $\left({ }^{\circ}\right)$ & 20.7 & 19.0 & 19.7 \\
\hline Improper angles $\left(^{\circ}\right)$ & 1.2 & 1.1 & 1.0 \\
\hline \multicolumn{4}{|l|}{ Residues (\%) in Ramachandran plot } \\
\hline Core region & 87.7 & 90.9 & 91.9 \\
\hline Additionally allowed region & 11.9 & 8.9 & 7.9 \\
\hline Generous allowed region & 0.4 & 0.2 & 0.2 \\
\hline Disallowed region & 0.0 & 0.0 & 0.0 \\
\hline
\end{tabular}

Values within parentheses refer to the last resolution shell.

${ }^{\text {a }} R_{\text {merge }}=\Sigma\left|\mathrm{I}_{\mathrm{i}}-\langle\mathrm{I}\rangle\right| / \Sigma\langle\mathrm{I}\rangle$

${ }^{\mathrm{b}} \mathrm{R}=\Sigma|| \mathrm{F}_{\mathrm{o}}|-| \mathrm{F}_{\mathrm{c}}|/ \Sigma| \mathrm{F}_{\mathrm{o}} \mid ; \mathrm{R}_{\text {free }}$ is calculated in the same way but for a subset of reflections that is not used in the refinement

sealing. A saturated solution of $\mathrm{K}_{2} \mathrm{CrO}_{4}$ was used instead for maintaining the crystal at r.h.88\%. Data collection commenced only $24 \mathrm{hr}$ after sealing the capillary to ensure equilibration. Intensity data from the native, the r.h.98\% and the r.h.88\% crystals were collected at room temperature $(293 \mathrm{~K})$ on a MAR imaging plate mounted on a Rigaku $\mathrm{Ru}-200$ rotating anode $\mathrm{X}$-ray generator, with a crystal-to-detector distance of 100 $\mathrm{mm}$. The data sets were processed and scaled in an identical manner using DENZO and SCALEPACK. ${ }^{15}$ The data collection statistics are summarized in Table I. The structure of native horse methemoglobin, obtained at high salt conditions, ${ }^{16}$ was used as the search model for molecular replacement. AMoRe ${ }^{17}$ calculations yielded best solutions with correlation coefficients of 46.6, 53.9, and $50.1 \%$ and R-factors $40.8,37.8$, and 39.2, respectively for data from the native, r.h.98\% and r.h.88\% crystals. The structures were refined in a similar manner using CNS incorporating the maximum likelihood target. ${ }^{18}$ In addition to those in the protein, the structural parameters of the heme group were also restrained. ${ }^{19}$ To start with, the structures were refined, first treating the whole tetrameric molecules as rigid bodies and then the subunits as rigid bodies. This was followed by cycles of refinement of positional parameters and temperature factors and model building using $\mathrm{O} .{ }^{20}$ Simulated annealing was also carried out in between the cycles. Identification of water oxygens was undertaken when $\mathrm{R}$ was in the $23-24 \%$ range. Initially, peaks greater than $3 \sigma$ in Fo-Fc maps and $1 \sigma$ in 2 Fo-Fc maps, were identified as corresponding to water oxygens. The limits were subsequently reduced to $2.5 \sigma$ and $0.8 \sigma$. Except in the case of the $\alpha 2$ subunit in the native structure, water oxygens coordinated to the heme iron could be identified in the first round itself. During most of the refinement cycles, noncrystallographic symmetry (NCS) constraints were applied separately to the $\alpha$ and $\beta$ subunits in each structure. The restraints were progressively removed and no NCS restraints were used in the final cycles. Bulk solvent corrections and anisotropic scaling were used throughout. The stereochemical acceptability of the structure was validated using PROCHECK. $^{21}$ The refinement parameters are listed in Table I. The final coordinates have been submitted to the Protein Data Bank (PDB references: native, $1 \mathrm{Y} 8 \mathrm{H}$; r.h.98\%, 1Y8I; r.h.88\%, 1Y8K). 
TABLE II. RMS Deviations (̊̊) in the Main-Chain Atoms Resulting From the Superposition of $\alpha 1 \beta 1$ Subunits of Pairs of Molecules ${ }^{\dagger}$

\begin{tabular}{rcccccccccc}
\hline & 1 & 2 & 3 & 4 & 5 & 6 & 7 & 8 & 9 & 10 \\
\hline 1 & - & 0.87 & 0.80 & 2.19 & 2.04 & 2.22 & 2.37 & 7.29 & 2.43 & 2.68 \\
2 & 0.30 & - & 0.53 & 1.73 & 1.58 & 1.88 & 1.89 & 6.82 & 2.78 & 2.15 \\
3 & 0.40 & 0.21 & - & 2.00 & 1.89 & 2.10 & 2.13 & 7.04 & 2.56 & 2.34 \\
4 & 0.42 & 0.27 & 0.31 & - & 0.41 & 1.37 & 0.82 & 5.62 & 4.34 & 0.97 \\
5 & 0.47 & 0.33 & 0.35 & 0.13 & - & 1.30 & 0.76 & 5.78 & 4.21 & 1.07 \\
6 & 0.52 & 0.33 & 0.33 & 0.16 & 0.19 & - & 1.13 & 5.50 & 4.27 & 1.52 \\
7 & 0.60 & 0.45 & 0.46 & 0.29 & 0.28 & 0.40 & - & 5.34 & 4.40 & 1.10 \\
8 & 0.90 & 0.84 & 0.89 & 0.78 & 0.79 & 0.85 & 0.82 & - & 9.22 & 5.20 \\
9 & 0.62 & 0.51 & 0.46 & 0.51 & 0.54 & 0.69 & 0.61 & 0.89 & - & 4.73 \\
10 & 0.73 & 0.58 & 0.55 & 0.49 & 0.46 & 0.67 & 0.50 & 0.86 & 0.44 & - \\
\hline
\end{tabular}

Those in the $\alpha 1 \beta 1$ subunits are given in the lower left and those in $\alpha 2 \beta 2$ subunits in the upper right.

1. Native; 2. r.h.98\%; 3. r.h.88\%; 4. Horse met (PDB code:1NS9); 5. Horse carbonmonoxy (1G0B); 6 . Horse met (1NS6); 7. Horse carbonmonoxybezafibrate complex (1IWH); 8. Horse deoxy (2DHB); 9. Human R2 (1BBB); 10. Human oxy (1HHO).

\section{RESULTS AND DISCUSSION Solvent Content and Quality of Diffraction Pattern}

Improvement in the quality of diffraction pattern associated with the reduction in solvent content was first demonstrated in this laboratory in monoclinic lysozyme. ${ }^{22-24}$ At that time it was perceived as, and it still is, an exception to the rule. In most cases, partial dehydration results in poorer quality of diffraction data. However, there have been a few instances where reduction in solvent has been made use of to obtain better quality data. ${ }^{25}$

In monoclinic lysozyme, the solvent content of the crystal was reduced by reducing environmental humidity. When the humidity is reduced below a threshold value, the crystal undergoes a structural transformation with loss of water. The solvent content reduces by about $10 \%$ to $23 \%$. The unit cell also changes with the addition of a twofold symmetry element which results in the two independent molecules in the native form becoming crystallographically equivalent. Thus in monoclinic lysozyme, the reduction in solvent content, which leads to better diffraction data, is accompanied by large structural changes.

The crystals of horse methemoglobin discussed here present an entirely different picture. There is hardly any noticeable structural change associated with loss of water. The difference in solvent content between the native and the $98 \%$ r.h. form is only $1.8 \%$. That between $98 \%$ and $88 \%$ forms is still lower at $1.2 \%$. However, each reduction is accompanied by significant improvement in the resolution. (Data quality decreases with further reduction of environmental humidity.) The reduction of solvent content necessary for improvement in data quality is among the lowest in the present case when compared to other protein crystals which exhibit this property. ${ }^{25}$ The structural basis for the improved resolution that accompany partial dehydration in some crystals, including those of horse methemoglobin reported here, is as yet unclear.

\section{The Quaternary Structure}

A major objective of the work is to explore the variability in the quaternary association of liganded horse hemoglobin.
The $R$ and $R 2$ states observed in the human protein provide a frame work for this exploration. The observation of states intermediate between the two in human methemogloin by the authors ${ }^{11}$ and in bovine carbonmonoxy hemoglobin by Mueser et al. ${ }^{12}$ adds a further dimension to the problem. The $\mathrm{R}$ state was observed in crystals grown at $\mathrm{pH} 6.7$ from a high salt medium ${ }^{4}$ while the R2 state was characterized in crystals grown at $\mathrm{pH} 5.8$ from a medium with low ionic strength. ${ }^{10}$ The crystals of human methemoglobin were grown at low ion concentration using PEG, a condition which has been suggested to favor the R2 state. On the other hand, the $\mathrm{pH}$ at which they were grown is similar to that used for preparing R-state human oxyhemoglobin. In the case of bovine carbonmonoxyhemoglobin, ${ }^{12}$ the low-salt/low $\mathrm{pH}$ form, crystallized under conditions that yield human R2 structure, the quaternary structure is intermediate between $R$ and $R 2$ states; the same is the case with crystals grown from a high salt medium at $\mathrm{pH} 7.2$, a condition which favors the $\mathrm{R}$ state in liganded human hemoglobin. However, the R2 state is exhibited by a low-salt/high $\mathrm{pH}$ form. These studies on human and bovine hemoglobin show that although liganded human and bovine hemoglobin exhibits the $\mathrm{R}$ state, the $\mathrm{R} 2$ state, and states intermediate between the two, the precise conditions for their occurrence are not yet clearly established.

As indicated above, the structural variability of the liganded state of two of the three most studied mammalian hemoglobin has been clearly established. The remaining one is that from horse. The structures of liganded horse hemoglobin available in the literature include those of aquomet and carbonmonoxyhemoglobin from crystals prepared in high salt conditions at $\mathrm{pH} 7.1^{14}$ and $\mathrm{pH} 8.5,{ }^{12}$ respectively. Crystals of hemichrome hemoglobin (pH 5.4) ${ }^{14}$ grown in the high salt conditions have also been analyzed. Yet another crystal analyzed is that of a complex of carbonmonoxyhemoglobin with bezafibrate, grown in lowsalt condition at $\mathrm{pH} 6.8 .^{26}$ The molecules in all these crystals are essentially in the $\mathrm{R}$ state. It is interesting to compare their structure with that of aquomethemoglobin obtained from the present analysis of crystals grown from a low-salt medium at $\mathrm{pH}$ 7.0. This has been done with the 


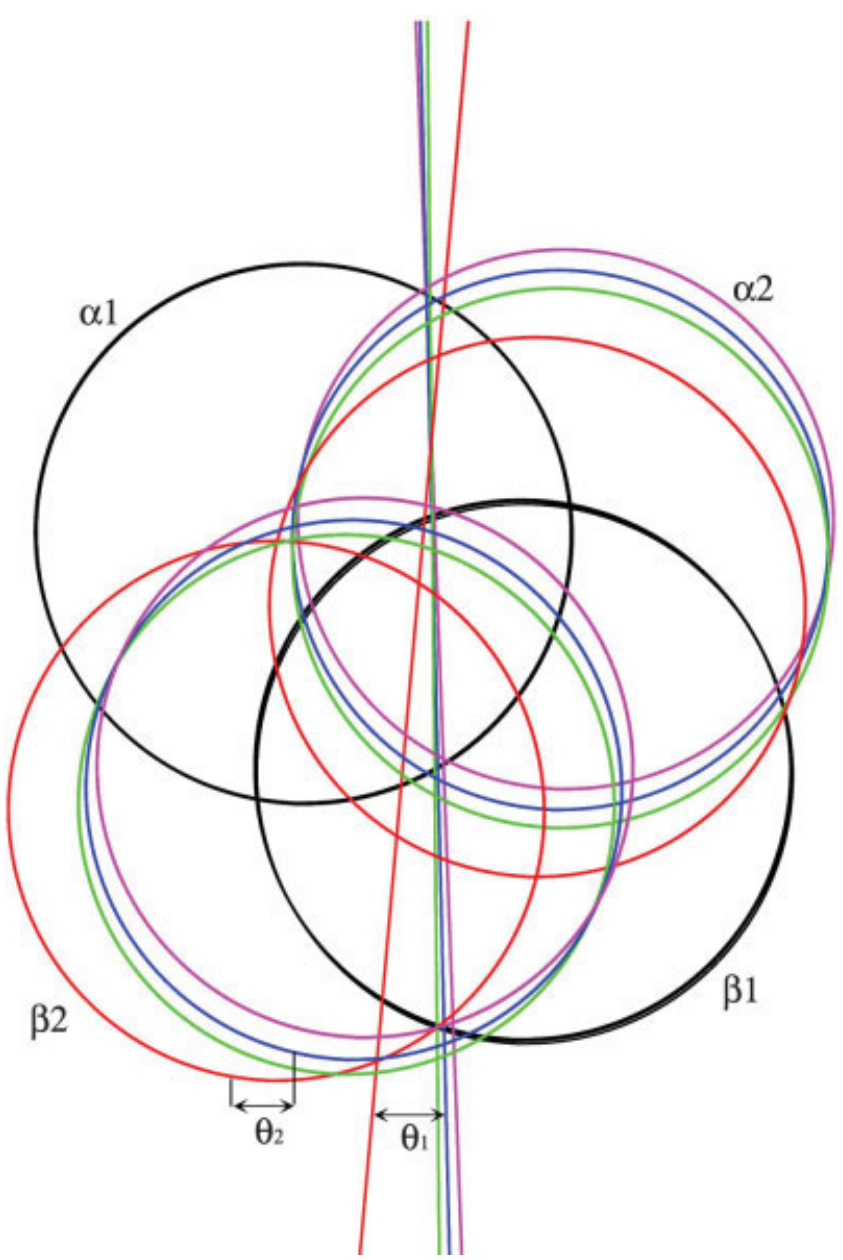

Fig. 1. Schematic diagram showing the orientation of $\alpha 2 \beta 2$ relative to $\alpha 1 \beta 1$ in the r.h.88\% structure reported here and other selected relevant structures. The $\alpha 1 \beta 1$ dimer in all the structures are superposed (black). The $\alpha 2 \beta 2$ dimer and the molecular dyad are shown in different colors: r.h. $88 \%$, blue; human R2, magenta; horse deoxy, red; horse met R, green. $\theta_{1}$ and $\theta_{2}$ values of all the relevant structures with respect to the r.h. $88 \%$ structure, are given in Table III. Please see text for details. This figure was generated using MOLSCRIPT. ${ }^{29}$

$\mathrm{R}$ and the $\mathrm{R} 2$ state structures of liganded human hemoglobin as the bench marks.

The asymmetric unit of the present structure contains a full tetrameric molecule while most other structures have only one $\alpha$ subunit and one $\beta$ subunit in the asymmetric unit. To facilitate comparison, the coordinates of the main-chain atoms of $\alpha 1 \beta 1$ and $\alpha 2 \beta 2$ in the tetramer were averaged about the molecular dyad, thus producing a tetramer with exact twofold symmetry as in other structures. The $\alpha 1 \beta 1$ subunits of different pairs of structures were then superposed using main-chain atoms. The resulting RMS deviations in $\alpha 1 \beta 1$ subunits and $\alpha 2 \beta 2$ subunits are listed in Table II. These calculations were performed using the computer program ALIGN. ${ }^{27}$ The deviations among $\alpha 1 \beta 1$ subunits provide a measure of the differences in the structure of the dimer; those in $\alpha 2 \beta 2$ largely depend upon the differences in the mutual orientation of the two dimers. Baldwin and Chothia ${ }^{28}$ have earlier shown that the change in the mutual orientation of $\alpha 1 \beta 1$ and $\alpha 2 \beta 2$ can
TABLE III. Angles $\left({ }^{\circ}\right) \theta_{1}$ (Upper Right) and $\theta_{2}$ (Lower Left) That Define the Change in Quaternary Structure Between Pairs of Structures

\begin{tabular}{rrccccccccc}
\hline & 1 & 2 & 3 & \multicolumn{1}{c}{4} & \multicolumn{1}{c}{5} & \multicolumn{1}{c}{6} & \multicolumn{1}{c}{7} & 8 & 9 & 10 \\
\hline 1 & - & 0.4 & 0.0 & 2.6 & 2.4 & 2.2 & 2.9 & 9.0 & 3.1 & 3.1 \\
2 & 0.9 & - & 0.4 & 2.1 & 1.9 & 1.7 & 2.4 & 8.7 & 3.6 & 2.6 \\
3 & 0.1 & 0.8 & - & 2.5 & 2.3 & 2.1 & 2.8 & 9.1 & 3.2 & 2.8 \\
4 & 5.2 & 4.3 & 5.0 & - & 0.0 & 0.8 & 0.7 & 6.7 & 5.7 & 0.5 \\
5 & 4.8 & 3.9 & 4.7 & 0.4 & - & 0.6 & 0.7 & 6.9 & 5.6 & 0.8 \\
6 & 4.4 & 3.4 & 4.2 & 1.5 & 1.3 & - & 0.8 & 6.9 & 5.3 & 1.3 \\
7 & 5.8 & 4.8 & 5.6 & 1.3 & 1.3 & 1.5 & - & 6.2 & 5.9 & 0.9 \\
8 & 18.1 & 17.3 & 18.1 & 13.5 & 13.9 & 13.7 & 12.4 & - & 12.1 & 6.6 \\
9 & 6.2 & 7.2 & 6.4 & 11.5 & 11.2 & 10.6 & 11.7 & 24.2 & - & 6.2 \\
10 & 6.1 & 5.2 & 5.7 & 1.1 & 1.7 & 2.6 & 1.9 & 13.1 & 12.4 & - \\
\hline
\end{tabular}

be described by angles $\theta_{1}$ and $\theta_{2}$ (Fig. 1) and a translation associated with $\theta_{2}$. The angles $\theta_{1}$ and $\theta_{2}$ between relevant pairs of structures are listed in Table III. The screw translations are small; their magnitude is about $1 \AA$ or less among liganded horse hemoglobin structures.

Tables II and III clearly show that the three horse methemoglobin structures with different solvent contents reported here, are in the same relaxed state. All the other relaxed horse hemoglobin structures are essentially in the $\mathrm{R}$ state; they and the liganded human $\mathrm{R}$-state structure together form a cluster. The structures reported here are nearly as different from the R2 structure as they are from the $\mathrm{R}$ structure. Tables II and III and Figure 1 clearly demonstrate that, in terms of quaternary structure, the horse methemoglobin structure presented here lies nearly halfway between the $\mathrm{R}$ and the $\mathrm{R} 2$ states.

In terms of inter-subunit interactions, the "switch region(s)" in the $\alpha 1 \beta 2-\alpha 2 \beta 1$ interfaces serves as an indicator of the quaternary state of the hemoglobin molecule. ${ }^{28}$ The geometry of this region in a few representative structures is illustrated in Figure 2. In the R state, His97 $\beta 2$ is located between Thr38 $\alpha 1$ and Thr $41 \alpha 1$, while in the $\mathrm{T}$ state the histidyl residue moves out of this region and is close only to $\mathrm{Thr} 41 \alpha 1$. In the R2 state also the histidyl residue is situated in between two threonyl residues, but it is farther away from them than in the $\mathrm{R}$ state. The appropriate distances involving the $\alpha$-carbon positions of the three residues in the relevant structures are listed in Table IV. These distances and Figure 2 also clearly show that the geometrical disposition of the three residues in the structures reported here are in between those in the $\mathrm{R}$ and the $\mathrm{R} 2$ states.

\section{CONCLUSION}

The present study leads to the characterization of a relaxed state other than the classical $\mathrm{R}$ state in horse hemoglobin. This state has geometrical features in between the $\mathrm{R}$ state and the R2 state described in human hemoglobin. This result, our earlier demonstration of the structural variability in the $R$ and $T$ states of human hemoglobin and the observation of the variability in the relaxed state of bovine hemoglobin in another study, together suggest that the relaxed as well as the tense hemoglobin molecules can access ensembles of states. 


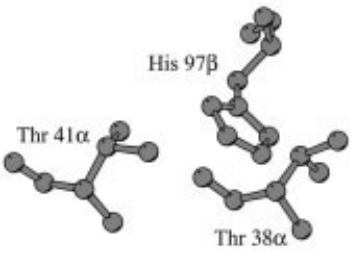

Horse R state
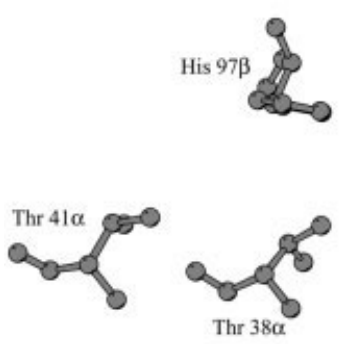

Human R2 state

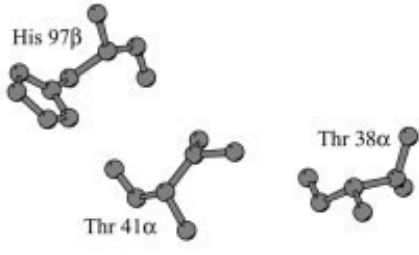

Horse T state
Fig. 2. Switch region in selected relevant structures. The appropriate distances in all the relevant structures are given in Table IV. This figure was generated using MOLSCRIPT. ${ }^{29}$

TABLE IV. Distances (̊̊) Between the $\mathbf{C}^{\alpha}$ Atoms of the Residues Involved in Switching Action ${ }^{\dagger}$

$\alpha 1 \beta 2$ interface

\begin{tabular}{lccc}
\cline { 3 - 4 } Structure & & His97ß2-Thr38 $\alpha 1$ & His97 $\beta 2-\mathrm{Th} 41 \alpha 1$ \\
\hline Native & 6.11 & 8.42 \\
r.h.98\% & 5.93 & 7.98 \\
r.h.88\% & 5.86 & 7.83 \\
Horse R & 1NS9 & 5.34 & 7.50 \\
& 1G0B & 5.37 & 7.78 \\
& 1NS6 & 5.29 & 7.38 \\
& 1IWH & 5.19 & 7.66 \\
Horse deoxy & 2DHB & - & 4.98 \\
Human R2 & 1BBB & 7.16 & 9.63 \\
Human oxy R & 1HHO & 5.19 & 7.28 \\
\hline
\end{tabular}

†The values have been averaged when two crystallographically independent sets are present.

\section{ACKNOWLEDGMENTS}

X-ray data were collected at the X-ray Facility for Structural Biology, supported by the Department of Science and Technology (DST) and the Department of Biotechnology (DBT). Computations were carried out at the Supercomputer Education and Research Centre at the Institute, and the Bioinformatics Centre and Graphics Facility (both supported by DBT). The work is funded by Council of Scientific and Industrial Research.

\section{REFERENCES}

1. Perutz MF. Stereochemistry of cooperative effects in haemoglobin. Nature 1970; 228:726-739.

2. Monod J, Wyman J, Changeux JP. On the nature of allosteric transitions: a plausible model. J Mol Biol 1965;12:88-118.

3. Tame JR. What is the true structure of liganded haemoglobin? Trends Biochem Sci. 1999;24:372-377.
4. Shaanan B. Structure of human oxyhaemoglobin at $2.1 \AA$ resolution. J Mol Biol 1983;171:31-59.

5. Fermi G, Perutz MF, Shaanan B, Fourme R. The crystal structure of human deoxyhaemoglobin at $1.74 \AA$ resolution. J Mol Biol 1984;175:159-174.

6. Baldwin JM. The structure of human carbonmonoxy haemoglobin at $2.7 \AA$ resolution. J Mol Biol 1980;136:103-128.

7. Vasquez GB, Ji X, Fronticelli C, Gilliland GL. Human carboxyhemoglobin at 2.2 ̊ resolution: Structure and solvent comparisons of R-state, R2-state, and T-state hemoglobins. Acta Crystallogr D Biol Crystallogr 1998;54:355-366.

8. Brzozowski A, Derewenda Z, Dodson E, Dodson G, Grabowski M, Liddington R, Skarzynski T, Vallely D. Bonding of molecular oxygen to T state human haemoglobin. Nature 1984;307:74-76.

9. Liddington R, Derewenda Z, Dodson E, Hubbard R, Dodson G. High resolution crystal structures and comparisons of deoxyhaemoglobin and two liganded T-state haemoglobins: T(alpha-oxy) haemoglobin and T(met) haemoglobin. J Mol Biol 1992;228:551-579.

10. Silva MM, Rogers PH, Arnone A. A third quaternary structure of human hemoglobin A at $1.7 \AA$ resolution. J Biol Chem 1992;267: 17248-17256.

11. Biswal BK, Vijayan M. Structure of human methaemoglobin: the variation of a theme. Curr Sci (India) 2001;81:1100-1105.

12. Mueser TC, Rogers PH, Arnone A. Interface sliding as illustrated by the multiple quaternary structures of liganded hemoglobin. Biochem 2000;39:15353-15364.

13. Biswal BK, Vijayan M. Structures of human oxy- and deoxy haemoglobin at different levels of humidity: variability in the $\mathrm{T}$ state. Acta Crystallogr D Biol Crystallogr 2002;58:1155-1161.

14. Robinson VL, Smith BB, Arnone A. A pH-dependent aquomet-tohemichrome transition in crystalline horse methemoglobin. Biochem 2003;42:10113-10125.

15. Otwinowski Z, Minor W. Processing of X-ray diffraction data collected in oscillation mode. Methods Enzymol 1997;276:307-326.

16. Ladner RC, Heidner EJ, Perutz MF. The structure of horse methaemoglobin at $2.0 \AA$ resolution. J Mol Biol 1977;114:385-414.

17. Navaza J. AMoRe: an automated package for molecular replacement. Acta Crystallogr A 1994;50:157-163.

18. Brunger AT, Adams PD, Clore GM, Delano WL, Gross P, GrosseKunstleve RW, Jiang J-S, Kuszewski J, Nilges M, Pannu NS, and others. Crystallography \& NMR system: a new software suite for macromolecular structure determination. Acta Crystallogr D 1998;54: 905-921.

19. Kuriyan J, Wilz S, Karplus M, Petsko GA. X-ray structure and refinement of carbon-monoxy(Fe II)-myoglobin at $1.5 \AA$ resolution. J Mol Biol 1986;192:133-154.

20. Jones TA, Zou J-Y, Cowan SW, Kjeldgaard M. Improved methods for building protein models in electron density maps and the location of errors in these models. Acta Crystallogr A 1991;47:110-119.

21. Laskowski RA, Moss DS, Thornton JM. Main-chain bond lengths and bond angles in protein structures. J Mol Biol 1993;231:1049-1067.

22. Salunke DM, Veerapandian B, Vijayan M. Water-mediated structural transformations in a new crystal form of ribonuclease A and tetragonal lysozyme. Curr Sci (India) 1984;53:231-235.

23. Salunke DM, Veerapandian B, Kodandapani R, Vijayan M. Watermediated transformations in protein crystals. Acta Crystallogr B 1985;41:431-436.

24. Madhusudan, Kodandapani R, Vijayan M. Protein hydration and water structure: X-ray analysis of a closely packed protein crystal with very low solvent content Acta Crystallogr D Biol Crystallogr 1993;49:234-245.

25. Heras B, Edeling MA, Byriel KA, Jones A, Raina S, Martin JL. Dehydration converts DsbG crystal diffraction from low to high resolution. Structure 2003;11:139-145.

26. Shibayama N, Miura S, Tame JRH, Yonetani T, Park S-Y. Crystal structure of horse carbonmonoxyhemoglobin-bezafibrate complex at 1.55-^̊ resolution. J Biol Chem 2002;277:38791-38796.

27. Cohen GE. ALIGN: a program to superimpose protein coordinates, accounting for insertions and deletions. J Appl Crystallogr 1997; 30:1160-1161.

28. Baldwin J, Chothia C. Haemoglobin: the structural changes related to ligand binding and its allosteric mechanism. J Mol Biol 1979;129:175-220.

29. Kraulis PJ. MOLSCRIPT: a program to produce both detailed and schematic plots of protein structures. J Appl Crystallogr 1991;24: 946-950. 